\title{
Cicatriz renal detectada mediante cintigrama renal DMSA en niños con primera pielonefritis aguda: estudio de factores de riesgo
}

\author{
Gilda Donoso $\mathbf{R}^{\mathbf{1}, 2}$, G abriel Lobo $\mathrm{S}^{\mathbf{1}, 2}$, Francisca Arnello $\mathbf{V}^{\mathbf{1}}$, \\ María Paz Arteaga V1 ${ }^{1}$ Claudia Coll $C^{1}$, Pilar Hevia J ${ }^{4}$, \\ Pía Rosati $M^{3}$, Elizabeth Lagos $R^{3}$, Carlos W olff $F^{2, a}$, \\ Andrés Pérez $R^{1, b}$, César Jiménez J ${ }^{1, b}$. \\ Renal scars after one year of follow \\ up in children with a first episode \\ of acute pyelonephritis
} \begin{abstract}
scar that eventually can lead to hypertension or renal failure. 99mTc-dimercaptosuccinic acid renal scintigraphy (RC DMSA) is a widely accepted technique to assess children with acute pyelonephritis. Aim: To evaluate the presence of residual kidney scars detected through RC DMSA, in children with a first episode of acute pyelonephritis. Patients and methods: Clinical records of children with a first episode of acute pyelonephritis that were assessed within seven days of the episode with RC DMSA were reviewed. Children were considered eligible if they did not have a new episode of acute pyelonephritis and a second RC DMSA, one year after the first episode, was performed. The presence or absence of a renal scar after one year was associated to demographic, scintigraphy and laboratory variables. Results: Fifty nine children, aged 1 month to 10 years, 35 females, were studied. Thirty nine percent had a renal scar in the scintigraphy perfomed after one year of follow up. The presence of a scar was correlated with a $\mathrm{C}$ reactive protein over $130 \mathrm{mg} / \mathrm{dl}$ and an altered relative renal function (below 44\%), during the acute phase. Conclusions: A high $\mathrm{C}$ reactive protein and alterations of relative renal function during the acute phase of acute pyelonephritis in children, may be risk factors for the development of renal scars in the long term follow up (Rev Méd Chile 2006; 134: 305-11). (Key w ords: C-reactive protein; Pyelonephritis; Radionuclide imaging)
\end{abstract}

Background: Acute pyelonephritis in children can leave a kidney

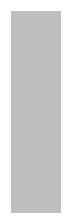

\footnotetext{
${ }^{a}$ Bioquímico

bTecnólogo Médico
}

Recibido el 22 de abril, 2005. Aceptado el 23 de agosto, 2005.

${ }^{1}$ Unidad de Medicina Nuclear, CDT-Hospital San Juan de Dios. ${ }^{2}$ Departamento de Medicina Occidente, Universidad de Chile. ${ }^{3}$ Servicio de Nefrología Infantil, Hospital San Juan de Dios. ${ }^{4}$ Departamento de Pediatría Occidente, Universidad de Chile.

Correspondencia a: Dra. Gilda Donoso Roselló. Unidad de Medicina Nuclear, CDT - Hospital San Juan de Dios. Av. Diego Portales 3239, Santiago. Fono: 4506869 Fax: 6815686. E mail: gdonosor@yahoo.com 
$\mathrm{L}$ a pielonefritis aguda (PNA) en niños tiene el riesgo de dejar cicatriz en el riñón, lo que con el tiempo puede llevar a hipertensión, insuficiencia renal o ambos $^{1}$. Estudios experimentales han demostrado que la misma respuesta inflamatoria aguda responsable de erradicar la bacteria es también la causante del daño al tejido renal y posterior cicatriz ${ }^{2,3}$

Es sabido que la cicatriz renal en infección urinaria ocurre sólo en sitios correspondientes a áreas de PNA previas. Por otra parte, en la mayoría de los casos, los procesos agudos renales son reversibles. Hasta hoy no se logra consenso sobre qué elementos serían los mejores predictores de daño renal permanente. Múltiples factores se han intentado asociar a la generación de cicatriz renal posterior a la pielonefritis aguda: virulencia del germen, retraso en el inicio del tratamiento antibiótico, edad del paciente, presencia de reflujo vésico ureteral (RVU), etc. Sin embargo, existe controversia sobre el rol de cada uno de ellos y el rol potencial de otros factores ${ }^{4-7}$.

Entre las técnicas aceptadas para el estudio de PNA, se encuentra el cintigrama renal con ácido dimercaptosuccínico (CR DMSA), que algunos grupos utilizan sólo para el seguimiento y otros también para el diagnóstico de los niños con esta enfermedad, ya que permite evaluar la progresión del daño renal y la pérdida funcional que se puede producir en el riñón afectado ${ }^{8}$. Un DMSA inicial anormal es probablemente el mejor elemento para identificar la población de riesgo de desarrollar cicatriz renal.

En este trabajo se evaluó la incidencia de cicatriz renal en nuestra población pediátrica con primera PNA y se analizó qué factores de la etapa aguda podrían ser indicadores de riesgo de desarrollar secuela renal.

\section{Pacientes y MÉTOdo}

Se revisaron las fichas clínicas de pacientes hospitalizados en el Servicio de Pediatría del Hospital San Juan de Dios, entre los años 1991 y 1999 con el diagnóstico clínico de primera $\mathrm{PNA}^{9-11}$, con urocultivo positivo obtenido con recuentos adecuados para las diferentes técnicas de recolección (>100.000 colonias/ml por recolector o por orina de $2^{\circ}$ chorro; $>10.000$ colonias $/ \mathrm{ml}$ por sondeo vesical o $>1$ colonias/ml por punción vesical), y CR DMSA alterado, realizado dentro de los 7 días posteriores al diagnóstico del episodio infeccioso.

A todos los pacientes se les practicó un CR DMSA de control 1 año después del episodio agudo. La ocurrencia de nuevos episodios de infecciones renales en ese período fue motivo de exclusión del estudio.

El CR DMSA fue realizado con técnica habitual en una Gamacámara Picker Dyna unida a un computador Alfa Nuclear, el que se utilizó para efectuar el cálculo de la función renal diferencial. La dosis de DMSA Tc ${ }^{99 m}$ se ajustó según la edad. Se obtuvieron imágenes entre las 2 y $4 \mathrm{~h}$ post inyección e.v. del trazador en proyecciones póstero anterior, oblicuas posteriores derecha e izquierda y ántero posterior.

El CR DMSA fue considerado anormal cuando presentaba una o más de las siguientes alteraciones: defecto de contraste focal único o múltiple, distribución irregular del trazador, disminución de la función relativa $(\leq 43 \%)$ y alteración del tamaño renal. El examen de control realizado en las mismas condiciones al año después del episodio agudo, determinó la presencia o no de cicatriz.

La existencia de RVU se estudió por uretrocistografía miccional radiológica o cistografía isotópica directa, clasificándolos en 2 grupos: leves-moderados y severos.

Los pacientes se clasificaron según edad en menores y mayores de 2 años, correlacionando ambos grupos con la presencia o no de cicatriz.

Se analizaron los parámetros de laboratorio: velocidad hemática de sedimentación (VHS), proteína $\mathrm{C}$ reactiva (PCR) y recuento de leucocitos en sangre así como el tamaño renal y la función renal relativa medidos en el CR DMSA obtenidos durante la fase aguda de la PN. Se consideró normal una PCR menor de $20 \mathrm{mg} / \mathrm{dl}$, una VHS hasta $30 \mathrm{~mm} / \mathrm{h}$ y leucocitos hasta 15.000 glóbulos blancos $/ \mathrm{mm}^{3}$. Se definió tres categorías para el tiempo de retraso en el inicio del tratamiento antibiótico (tiempo transcurrido entre el inicio de la fiebre y el inicio de la terapia antibiótica): hasta $72 \mathrm{~h}$, entre 3 y 5 días y más de 5 días.

Se excluyeron de este estudio los pacientes con malformaciones congénitas, excepto RVU.

Estadística. Se utilizó la prueba de Chi cuadrado, y prueba "t" de Student si la distribución de valores 
era normal. Se consideró como nivel de significancia estadística un valor de $\mathrm{p}<0,05$. Se realizó además análisis ROC (Receiver Operating Characteristic) de los parámetros que presentaron correlación significativa con la presencia de cicatriz.

\section{Resultados}

Durante el período de estudio 59 pacientes cumplieron los criterios de inclusión; 35 fueron mujeres (59\%). La mediana de edad fue de 9 meses (intervalo: 1 mes-10 años), 45 pacientes tenían menos de 2 años (76\%).

El 39\% de la población estudiada tenía cicatriz renal al año del episodio infeccioso agudo (Figura 1).

No hubo diferencias significativas en relación a sexo y edad entre el grupo que quedó con cicatriz y el que no la presentó (p: 0,534 y p: 0,979, respectivamente). No se encontró diferencias significativas ( $\mathrm{p}>0,478)$ al correlacionar la presencia de cicatriz renal con el tiempo entre el diagnóstico de PNA y el inicio de tratamiento.

En la Tabla 1 se muestra los resultados de los parámetros de laboratorio obtenidos durante la fase aguda de la PNA - VHS, PCR y recuento de leucocitos según la presencia o no de cicatriz renal, existiendo sólo diferencia estadísticamente significativa en la PCR.

Se demostró RVU en 16 de 56 pacientes en que se realizó el estudio (29\%), 8 de ellos con cicatriz renal y de los 41 sin RVU, 15 tuvieron cicatriz, no encontrándose diferencias significativas entre estos grupos ( $p>0,05)$. Tampoco se obtuvo diferencias significativas al agrupar pacientes, según grado de RVU y presencia de cicatriz ( $p>0,05)$.

El tamaño renal no se asoció a la existencia de cicatriz ( $p>0,05)$.

La función renal relativa mostró diferencia significativa entre los pacientes con y sin cicatriz renal (Tabla 2).

El análisis de curvas ROC de la PCR y la función renal relativa, determinó que los puntos de corte para diferenciar los pacientes que se asocian o no a cicatriz renal fue $>130 \mathrm{mg} / \mathrm{dl}$ para la PCR y $\leq 43 \%$ en la función renal relativa (Figura 2).

La sensibilidad y la especificidad del PCR (para un punto de corte $>130 \mathrm{mg} / \mathrm{dl}$ ) encontradas con este método son:

Sensibilidad: $\quad 68,2 \%$

Especificidad: $\quad 68,7 \%$

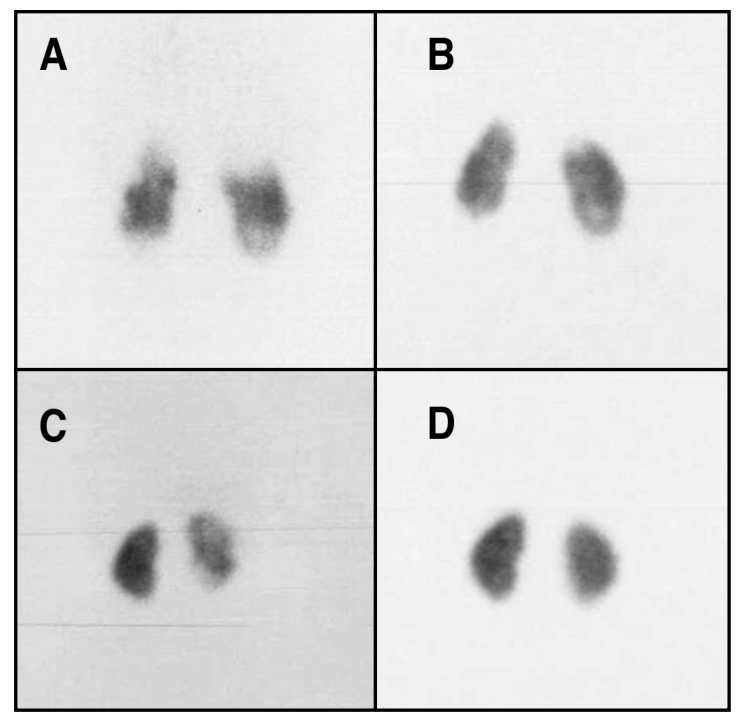

Figura 1. Cintigrama renal DMSA en fase aguda de primera PN y control al año, proyección póstero anterior. 1a) Alteraciones renales bilaterales en la fase aguda. 1b) Persistencia de alteraciones renales bilaterales, mayor a derecha en control tardío, correspondientes a cicatrices. 2a) Alteraciones de riñón derecho en fase aguda. 2b) Regresión completa de las alteraciones en el control del año. 


\section{Tabla 1. Resultados obtenidos en diferentes parámetros de laboratorio estudiados según la presencia de cicatriz renal}

\begin{tabular}{|lccc|}
\hline & $\begin{array}{c}\text { Sin cicatriz } \\
\text { (promedio } \pm \text { DS) }\end{array}$ & $\begin{array}{c}\text { Con cicatriz } \\
\text { (promedio } \pm \text { DS) }\end{array}$ & $\mathrm{p}$ \\
\hline PCR & $110,18 \pm 70,33$ & $155,6 \pm 74,04$ & $0,021^{*}$ \\
VHS & $74,94 \pm 33,17$ & $84,15 \pm 33,32$ & 0,303 \\
Leucocitos & $20.763 \pm 7.980$ & $19.582 \pm 7.244$ & 0,568 \\
\hline
\end{tabular}

*Estadísticamente significativo (prueba t de Student).

Tabla 2. Relación entre función renal relativa en el cintigrama renal en la fase aguda de una primera pielonefritis y cicatriz renal

\begin{tabular}{|lcc|}
\hline & Sin cicatriz & Con cicatriz \\
\hline Función renal normal & 31 & 13 \\
Función renal disminuida & 5 & 10 \\
\hline
\end{tabular}

Prueba Chi cuadrado: $\mathrm{p}=0,025$ (estadísticamente significativo).

Para la función relativa (punto de corte $\leq 43 \%$ ), estas son:

Sensibilidad: $\quad 43,5 \%$

Especificidad: $\quad 86,1 \%$

Se encontró diferencias estadísticamente significativas entre el grupo que quedó con cicatriz del que no lo hizo al considerar pacientes con PCR $>130$ $\mathrm{mg} /$ dl y función relativa $\leq 43 \%$ (Tabla 3). Considerando los criterios de cortes señalados, la sensibilidad fue $29 \%$, la especificidad $97 \%$, el valor predictivo positivo $14 \%$ y valor predictivo negativo $67 \%$. El 46\% de los pacientes estudiados tenían sólo la PCR $>130 \mathrm{mg} / \mathrm{dl} ; 22 \%$ tenía sólo la función relativa disminuida y $12 \%$ cumplía ambas condiciones.

El $65 \%$ de los pacientes con cicatriz renal tenían sólo PCR >130 mg/dl (15/23); 43\% sólo la función renal relativa disminuida y $26 \%$ cumplía ambas condiciones.

No se encontró diferencias significativas entre los pacientes con y sin cicatriz y valores de PCR mayor o menor a $130 \mathrm{mg} / \mathrm{dl}$ (p: 0,39).
Tabla 3. Relación entre PC R y función renal relativa en la fase aguda de una primera pielonefritis $\mathrm{v} / \mathrm{s}$ cicatriz renal

\begin{tabular}{|lcc|}
\hline & Sin cicatriz & Con cicatriz \\
\hline $\begin{array}{l}\text { PCR }>130 \mathrm{mg} / \mathrm{dl} \mathrm{y} \\
\text { función relativa } \leq 43 \% \\
\mathrm{PCR}<130 \mathrm{mg} / \mathrm{dl} \mathrm{y} \\
\text { función relativa }>43 \%\end{array}$ & 1 & 6 \\
\hline
\end{tabular}

Prueba Chi cuadrado: $\mathrm{p}=0,003$.

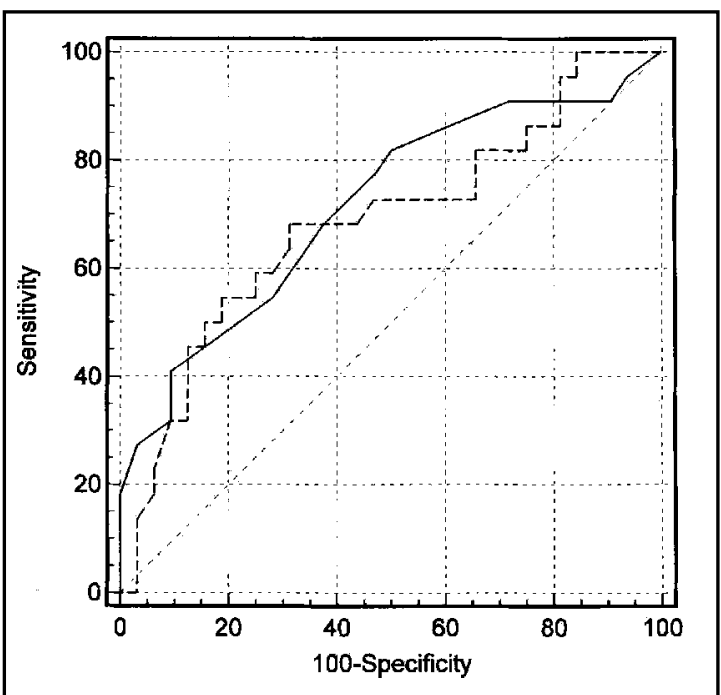

Figura 2. Curva de análisis ROC. Área bajo la curva ROC de función relativa $=0,687$. Área bajo la curva ROC de PCR=0,685. 
DISCUSIÓN

Múltiples factores de riesgo como menor edad, alto grado de RVU, virulencia del microorganismo causante y retraso en el inicio del tratamiento antibiótico, han sido correlacionados con el desarrollo de cicatriz renal, sin embargo, la patogénesis de ella es aún muy controvertida ${ }^{4,12-14}$.

El 39\% de los pacientes mostró cicatriz renal al año del episodio agudo de PN, cifra semejante a la encontrada por otros grupos, tanto extranjeros como nacionales $7,12,13$, lo que hace poco probable que las características socioculturales o económicas puedan influir.

Estudios experimentales y clínicos ${ }^{4,6}$ han demostrado que la cicatriz renal siempre se desarrolla en el mismo sitio en que ocurrió la infección inicial y es este grupo de pacientes con cicatriz renal el que tiene riesgo de desarrollar hipertensión arterial, insuficiencia renal o ambos. Estudios nacionales muestran que en $17 \%$ de los pacientes con insuficiencia renal crónica, ésta es debida a nefropatía por reflujo y pielonefritis ${ }^{15}$.

Para mantener una vigilancia médica más estricta, es necesario identificar la población de riesgo de desarrollar una cicatriz renal. Este grupo estaría formado por aquellos pacientes que han presentado compromiso renal durante el episodio agudo de la infección urinaria y, para este efecto, está ampliamente aceptado el uso del CR DMSA $8,16,17$.

Además de identificar los pacientes con compromiso renal durante la fase aguda de la infección urinaria, existe amplio consenso en el uso del CR DMSA para la detección de cicatrices renales ${ }^{6,8}$. En lo que aún existe controversia es en el momento adecuado para realizar el estudio control. La literatura sugiere que 6 meses es el tiempo requerido para definir la existencia de cicatriz, pero existe discusión ya que hay descritos porcentajes variables de pacientes que muestran mejoría completa entre los 6 meses y el año de ocurrida la PNA ${ }^{2,13,18}$. Considerando esto último, en nuestro estudio definimos la presencia o no de cicatriz al año del episodio agudo, sin que existieran nuevos cuadros infecciosos en ese período. Sin embargo, es necesario un mayor seguimiento para precisar mejor el momento oportuno para hacer el control, ya que evidentemente existe mayor porcentaje de pérdida de pacientes a mayor tiempo de seguimiento.
Se ha sugerido que a menor edad habría más riesgo de desarrollar cicatriz renal ${ }^{5,19}$, lo que no fue demostrado en este estudio, así como tampoco se encontró diferencias significativas entre hombres y mujeres en la predisposición a desarrollar cicatriz renal. Estos hallazgos concuerdan con lo señalado por otros grupos 5 y permite concluir que todos los niños con PNA son de riesgo y serán beneficiados por todas las medidas de prevención y control.

Tampoco encontramos diferencias significativas entre aquellos pacientes que comenzaron tratamiento con tiempos de retraso diferentes, lo que ya ha sido descrito y aún es discutido ${ }^{2,20}$. No existen estudios que muestren exactamente cuánto tiempo de retraso en el inicio de la terapia es realmente de riesgo para el riñón, por lo que frente al diagnóstico de PNA, la terapia debe iniciarse rápidamente.

La presencia o no de RVU tampoco se asoció a daño renal permanente, siendo coincidente con lo descrito en la literatura actual en que existe el consenso de que el compromiso renal en la PNA no se relaciona con la presencia de RVU. Se ha descrito que en niños con RVU existe mayor posibilidad de daño renal cuando el reflujo es severo ${ }^{14,21}$, sin embargo esto no fue encontrado en la muestra de pacientes estudiados, quizás debido al bajo número de reflujos positivos.

En este estudio se pudo determinar que sólo la PCR se relaciona con el desarrollo de cicatriz pero debe considerarse lo inespecífico que es este parámetro ${ }^{22}$, ya que cualquier patología infecciosa lo puede alterar y, además, tanto el grupo que quedó con cicatriz como el que no, tiene valores de PCR sobre los valores normales. Empleando el análisis de curvas ROC, se pudo determinar que el punto de corte para los valores de PCR que se asocian con presencia de cicatriz fue $130 \mathrm{mg} / \mathrm{dl}$, evidenciándose con este método también la baja sensibilidad y especificidad de este factor $(68,2 \mathrm{y}$ $68,7 \%$, respectivamente). El $46 \%$ del total de pacientes y $65 \%$ de los que quedaron con secuela tuvieron la PCR >130 mg/dl. Esto permite considerar que este parámetro por sí solo no puede ser usado con seguridad como indicador de riesgo de desarrollar daño renal.

Entre los parámetros cintigráficos analizados en el CR DMSA inicial, una menor función relativa se asoció en forma significativa a la existencia de 
cicatriz renal en el control al año. Este hallazgo es ratificado por los resultados obtenidos en el análisis ROC, en que el mejor punto de corte sería una función relativa menor o igual a $43 \%$, valor que aplicamos en nuestro servicio en forma rutinaria para definir una disminución de función. Este mismo análisis nos permite ver además, que la sensibilidad relacionada a la presencia de cicatriz es baja, siendo mejor su especificidad. La menor función relativa asociada a la presencia de cicatriz renal puede explicarse porque en el CR DMSA las zonas de hipocaptación o menor contraste, son secundarias a la isquemia y daño tubular provocados por la infección, por lo que a mayor gravedad, más zonas hipocaptantes, lo que se traducirá también en una disminución de la función relativa. Si el compromiso infeccioso durante la fase aguda es importante, se puede pensar que hay más riesgo de desarrollar secuelas.

Es importante notar que se excluyó el CR cuando tenía sólo la función relativa disminuida, ya que así no se incluyeron riñones pequeños, hipoplásicos congénitos que van a tener función relativa disminuida sólo por su menor tamaño, sin asociarse necesariamente a una PNA.

Algunos estudios intentan correlacionar el grado de captación del DMSA en la PNA con el riesgo de desarrollar cicatriz renal, utilizando diferentes parámetros, algunos de ellos cuantitativos en que se evalúa la extensión y la intensidad del compro-

\section{REFERENCIAS}

1. Jacobson SH, Eklof O, ERIKSson CG, Lins L-E, Tidgren B, Winberg J. Development of hypertension and uraemia after pyelonephritis in childhood: 27 years follow up. BMJ 1989; 299: 703-6.

2. Rushton HG, Majd M. Dimercaptosuccinic acid renal scintigraphy for the evaluation of pyelonephritis and scarring: a review of experimental and clinical studies. J Urol 1992; 148: 1726-32.

3. Allen TD, Arant BS Jr, Roberts JA. Commentary: vesicoureteral reflux -1992. J Urol 1992; 148: 1758-60.

4. Jakobsson B, Berg V, Svensson L. Renal scar after acute pyelonephritis. Arch Dis Child 1994; 70: 111-15. miso cortical ${ }^{21,23}$. Existen diferentes parámetros cintigráficos susceptibles de analizar y son necesarios más estudios de seguimiento para validarlos.

Creemos importante destacar que al considerar una PCR $>130 \mathrm{mg} / \mathrm{dl}$ junto a una función renal relativa $\leq 43 \%$, aunque se obtiene una baja sensibilidad en cambio se obtiene una alta especificidad (97\%) para identificar quiénes tendrán cicatriz renal, lo que creemos importante ya que podría orientar con mayor seguridad el tratamiento y seguimiento de los pacientes que desarrollarán cicatriz.

En conclusión, considerando que mundialmente la prevención y el manejo de las cicatrices renales es un tema controvertido, intentamos desarrollar una estrategia para identificar niños en riesgo de desarrollar daño renal progresivo. En esta línea, es útil realizar un CR DMSA en la fase aguda, con determinación de la función renal relativa y luego un control alejado para determinar la presencia de cicatriz. El tiempo necesario para realizar este control alejado es aún controversial, siendo los 6 meses para algunos y un año para otros. Según nuestros resultados la edad, sexo, VHS y leucocitosis en la fase aguda de la PN no sirven para sospechar la posibilidad de desarrollar una cicatriz renal, sólo la PCR, mayor de $130 \mathrm{mg} / \mathrm{dl}$, junto a una función renal relativa menor o igual a $43 \%$ medida en el CR DMSA inicial, podrían ser considerados factores de riesgo debido a la alta especificidad de estos parámetros.

5. Benador D, Benador N, Slosman D, Mermillod B, GIRADIN E. Are younger children at highest risk of renal sequelae after pyelonephritis? Lancet 1997; 349: 17-19.

6. Rushton HG, Majd M, Jantausch B, Wiederman BL, Belman B. Renal scarring following reflux and nonreflux pyelonephritis in children; evaluation with 99m Technetium Dimercaptosuccinic acid scintigraphy. J Urol 1992; 147: 1327-32.

7. Gordon I. Vesico-ureteric reflux, urinary- tract infection and renal damage in children. Lancet 1995; 346: 489-90.

8. Piepsz A, Blaufox Md, Gordon I, Granerus G, Majd M, O'reilly P ET al. Consensus on renal cortical 
scintigraphy in children with urinary tract infection. Sem Nucl Med 1999; XXIX: 160-74.

9. JodAl U, LindBERG U, Lincoln K. Level diagnosis of symptomatic urinary tract infections in childhood. Acta Paediatr Scand 1975; 64: 201-8.

10. Practice parameter: the diagnosis, treatment and the evaluation of the initial urinary tract infection in febrile infants and young children. American Academy of Pediatrics. Committee on Quality Improvement. Subcommittee on urinary tract infections. Pediatrics 1999; 103: 843-52.

11. Rushton HG, MD, FAAP. Urinary tract infections in children. Epidemiology, evaluation and management. Pediatric Clinics of North America 1997; 44: 1133-68.

12. Jakobsson B, Jacobsson SH, HJalmar K. Vesicoureteric reflux and other risk factors for renal damage: identification of high and low risk children. Acta Paediatr Suppl 1999; 431: 31-9.

13. Orellana P, Cavagnaro F, Baquedano P, Lagomarsino E, García C, Villarroel l et al. Factores de riesgo de daño renal permanente en niños con infección del tracto urinario. Rev Méd Chile 2002; 130: 1147-53.

14. Ditchfield M, De Campo J, Cook D, Nolan T, Powell H, Sloane R et al. Vesicoureteral reflux: an accurate predictor of acute pyelonephritis in childhood urinary tract infection? Radiology 1994; 190: 413-5.

15. Lagomarsino E, Valenzuela A, Cavagnaro F, Solar E. Chronic renal failure in pediatrics 1996. Chilean survey. Pediatr Nephrol 1999; 13: 288-91.

16. Stokland E, Hellstrom M, Jacobson B, Jodal V, Lundgren P Sixt R. Early Tc99m dimercaptosucci- nic acid (DMSA) in symptomatic first time urinary infection. Paediatrics 1996; 85: 430-6.

17. Donoso G, Lobo G, Arnello F, Arteaga MP, Hevia P, Rosati P ET al. Cintigrama renal DMSA en niños con primera pielonefritis aguda: correlación con exámenes de laboratorio, ecografía y la presencia de reflujo vésicoureteral. Rev Méd Chile 2004; 132: 58-64.

18. Jakobsson B, Svensson L. Transient pyelonephritic changes on $99 \mathrm{~m}$ technetium- dimercaptosuccinic acid scan at least five months after infection. Acta Pediatr 1997; 86: 803-7.

19. Vernon SJ, Coulthard MG, Lamber J, Kein MJ, MATHEws JNS. New renal scarring in children who at age 3 and 4 years had had normal scans with dimercaptosuccinic acid: follow up studies. BMJ 1997; 315: 905-8.

20. Levtchenko E, lahy C, Levy L, Ham H, Piepsz A. Treatment of children with acute pyelonephritis: a prospective randomized study. Pediatr Nephrol 2001; 16: 878-84.

21. Biggi A, Dardanelli l, Cussino P, Pomero G, Noello CH, Sernia O et al. Prognostic value of the acute DMSA scan in children with first urinary tract infection. Pediatr Nephrol 2001; 16: 800-4.

22. Herrera P, Duffau T. Existen bases para el uso de la proteína $C$ reactiva en la detección de infecciones bacterianas en niños? Rev Méd Chile 2005; 133: 541-6.

23. Hitzel A, Liard A, Dacher J-N, Gardin I, Ménard J-F, ManRique A ET AL. Quantitative analysis of Tc99mDMSA during acute pyelonephritis for prediction of long-term renal scarring. J Nucl Med 2004; 45: 285-9. 